\title{
A Analysis of Raindrop Diameters for Rainfall Attenuation in Southern Africa
}

\author{
O. Adetan ${ }^{*}$, O.O. Obiyemi** \\ * Department of Electrical and Electronic Engineering, Ekiti State University Ado Ekiti, Nigeria \\ **Department of Electrical and Electronic Engineering, Osun State University, Osogbo, Nigeria
}

\section{Article Info \\ Article history: \\ Received Aug 6, 2015 \\ Revised Oct 12, 2015 \\ Accepted Nov 6, 2015}

\section{Keyword:}

Drop size distribution

Gamma model

Lognormal model

Raindrop critical diameters

Specific rainfall attenuation

\begin{abstract}
The influence of critical raindrop diameters on the specific rainfall attenuation in Durban $\left(29^{\circ} 52^{\prime} \mathrm{S}, 30^{\circ} 58^{\prime} \mathrm{E}\right)$, South Africa using various rainfall regimes is analyzed in this paper. Different rain rate values representing drizzle, widespread, shower and thunderstorm are selected for the purpose of analysis over the measured raindrop size distribution. The three-parameter lognormal and gamma DSD models with shape parameter of 2 are used to estimate the parameters required to investigate the drop sizes which produce a major contribution to the total specific rainfall attenuation for the selected rain rate values. The computed total specific attenuation increases with increasing frequencies and rain rates. The highest and prevailing contribution to the specific attenuation occurs at $D \sim 2 \mathrm{~mm}$ for the stratiform (drizzle or widespread) and convective (shower or thunderstorm) rain types for the models considered. The total percentage fraction formed by drops in the diameter range $0.5 \mathrm{~mm} \leq D \leq 2.5 \mathrm{~mm}$ and $1.0 \mathrm{~mm} \leq D \leq 3.0 \mathrm{mmare}$ found to be most critical for the specific rain attenuation for the stratiform (drizzle and widespread) and convective (shower and thunderstorm) rainfall types especially at higher frequencies.
\end{abstract}

Copyright $(2016$ Institute of Advanced Engineering and Science. All rights reserved.

\section{Corresponding Author:}

O. Adetan,

Department of Electrical and Electronic Engineering,

Ekiti State University,

Ado Ekiti, Nigeria.

Email: oadetan@gmail.com

\section{INTRODUCTION}

Rain has been identified as one of the major and important parameters affecting the propagation of signals in the microwave (3-30 GHz) and millimeter $(30-300 \mathrm{GHz})$ wave bands. Other rain factors such as the canting angle, drop size and raindrop shapes also have profound effects on waves propagating in these bands at extremely high frequencies [1]. The specific rainfall attenuation is often predicted from three parameters, which are; the frequency, rain rate and polarization, where the population of the raindrops is represented by the single parameter, rainfall rate [2]. Although the rainfall rate remains very useful and has been broadly used for rain attenuation prediction [3], [4] and [5], a good knowledge of the drop size distribution (DSD) is very essential in the estimation of the rainfall attenuation at these radio frequency bands because it governs all the microwave and rainfall integral relations. The modeling of the DSD varies from one climate to another. Drop size distribution modeling in temperate region; characterized by moderate rainfall is well suitable with models such as proposed by Marshall and Palmer [6], Laws and Parsons [7] and the negative exponential model of gamma [8].

The modeling of DSD in the tropical region is most suitable with the globally accepted Ajayi and Olsen [9] lognormal model. In Durban, South Africa, a reasonable number of works have been carried out on rainfall attenuation and DSD [10], [11], [12], [13], [14] and [15] establishing the suitability of the lognormal 
and gamma models for DSD modeling in the region. Similarly, various approaches and models have been adopted by some researchers across the globe to investigate the particular contributions of certain raindrop diameters to the specific rain attenuation [16], [17], [18], [19] and [20]. The influence of critical raindrop diameters on the specific rainfall attenuation in Durban, South Africa using various rainfall regimes is analyzed in this work. Different rain rate values representing drizzle (below $5 \mathrm{~mm} / \mathrm{h}$ ), widespread $(5-20$ $\mathrm{mm} / \mathrm{h}$ ) shower $(20-50 \mathrm{~mm} / \mathrm{h})$ and thunderstorm (above $50 \mathrm{~mm} / \mathrm{h}$ ) as classified according to [9] and [21] are selected for the purpose of analysis over the measured raindrop size distribution. The three-parameter lognormal and gamma DSD models with shape parameter of 2 as determined in [15] for Durban, South Africa are used to represent the measured DSD, N(D).

\section{DISDROMETER DATA ANALYSIS}

The Joss-Waldvogel (J-W) RD-80 [22] disdrometer installed in 2008 at the rooftop of the School of Electrical, Electronic and Computer Engineering, University of KwaZulu-Natal was used to obtain over 80, 000 data samples for this work. The disdrometer converts the momentum of each falling drop impacting on the sensor's surface into an electric pulse of commensurate voltage. The detectable diameter range is divided into 20 intervals. The sampling time, $T$ of the disdrometer is $60 \mathrm{~s}$ with the sampling area, $S$ of $50 \mathrm{~cm}^{2}(0.005$ $\mathrm{m}^{2}$ ). The data was gathered over a period of three (3) years. The data was sorted and classified into different types of rain based on rainfall rates $R(\mathrm{~mm} / \mathrm{h})$ as classified in [6] and [18] namely: drizzle, widespread, shower and thunderstorm. The minimum and maximum rainfall rates were $0.003 \mathrm{~mm} / \mathrm{h}$ and $117.15 \mathrm{~mm} / \mathrm{h}$ respectively. Rainfall events with overall sum of drops less than 10 were ignored from the disdrometer data to compensate for the dead-time errors. The instrument is located at an altitude of 140 meters above sea level. The location site is free of noise and protected from very strong winds. Equipment outage was observed but very minimal. From the disdrometer measurement, $N\left(D_{i}\right)$ is calculated as [22]:

$$
N\left(D_{i}\right)=\frac{n_{i}}{v\left(D_{i}\right) * S * T * d D_{i}}
$$

where $N\left(D_{i}\right)$ is the number of drops measured in the dropsize class, $n_{i}$ is the number of drops per channel, $v(D)$ is the Gun-Kinzer [23] terminal velocity of water droplets and $d D_{i}$ is the change in diameter of the (channel) in $\mathrm{mm}$.

\section{DROP SSIZE DISTRIBUTION MODELS}

Raindrop size distributions were used to estimate the specific rainfall attenuation. Two DSD models are considered in this work; the lognormal and the gamma DSD models.

\subsection{Lognormal DSD Model}

The lognormal distribution model is expressed by [9] and [21]:

$$
N(D)=\frac{N_{T}}{\sqrt{2 \pi} \times \sigma \times D} \exp \left[-\frac{1}{2}\left(\frac{\ln (D)-\mu}{\sigma}\right)^{2}\right]
$$

where $N_{T}$ (concentration of rainfall drops) is a function of climate, geographical location of measurements and rainfall type, $\mu$ is the mean of $\ln (D)$ and $\sigma$ is the standard deviation which determines the width of the distribution. The three parameters in (2) above are related to the rainfall rate $R$ by [9]:

$$
\begin{aligned}
& N_{T}=a_{0} R^{b_{0}} \\
& \mu=A_{\mu}+B_{\mu} \ln R \\
& \sigma^{2}=A_{\sigma}+B_{\sigma} \ln R
\end{aligned}
$$

where $a_{0}, b_{0}, A_{\mu}, B_{\mu}, A_{\sigma}$ and $B_{\sigma}$ are coefficients of moment regression determined using the least squares method of regression technique. The three-parameter lognormal DSD model as determined in [15] is given as:

$$
\mu=-0.3104+0.1331 \ln R
$$




$$
\begin{aligned}
& \sigma^{2}=0.0738+0.0099 \ln R \\
& N_{T}=268.07 R^{0.4068}
\end{aligned}
$$

\subsection{Gamma DSD Model}

The three-parameter gamma distribution model in Durban as expressed by Tokay and Short [24] in the form of (2) was studied by Adetan and Afullo [15] with $N_{o}\left(\mathrm{~m}^{-3} \mathrm{~mm}^{-1-\mu}\right)$ indicating the scaling parameter, $\mu$ (unitless) is the shape parameter, and $\Lambda$ is the slope parameter in $\mathrm{mm}^{-1}$. While the shape parameter does influence the slope of the distribution at larger diameter bound, it contributes largely on the curvature of the distribution at small diameters. The gamma distribution is particularly useful in tropical regions where the exponential distribution was found to be inadequate [9] and [24].

$$
N(D)=N_{o} * D_{i}{ }^{\mu} \exp \left(-\Lambda D_{i}\right)
$$

where $N_{o}=78259 R^{-0.156}, \Lambda=6.3209 R^{-0.168}$ and $\mu=2$

\section{THE SPECIFIC RAINFALL ATTENUATION AND THE EXTINCTION CROSS SECTION}

Generally, the specific rainfall attenuation $\gamma(\mathrm{dB} / \mathrm{km})$ is given by the relation [25]:

$\gamma=4.343 \times 10^{-3} \int_{0}^{D_{\max }} Q_{t} \cdot N(D) d D \quad[d B / k m]$

where $Q_{t}$ is the total extinction cross-section, which is a function of the drop diameter, $D$, the wavelength, $\lambda$ and the complex refractivity index of water drop, $m$ ( which depends on the frequency, $f$ and the temperature, $T$ ). The extinction cross section, $Q_{t}$ is evaluated using (11) as provided by Odedina and Afullo [13] in a power law relation, where $\kappa$ and $\alpha$ are the coefficients that depend on rain rate, temperature, polarization and canting angle of droplets.

$$
Q_{t}=\kappa\left(\frac{D}{2}\right)^{\alpha}
$$

The classical scattering theory of Mie [26], [27] is used to compute the values of $\kappa$ and $\alpha$ while assuming that each spherical raindrop illuminated by a plane wave is uniformly distributed in a rain field medium. The distance between each drop is assumed large enough to avoid collision. Table 1 shows the values of $\kappa$ and $\alpha$ at $f=5-100 \mathrm{GHz}$. The total rainfall attenuation therefore, is evaluated by integrating over all the raindrop sizes.

$$
\gamma=4.343 \times 10^{-3} \int_{d_{1}}^{d_{\max }} N(D) * \kappa\left(\frac{D_{i}}{2}\right)^{\alpha}
$$

Table 1. Values of $\kappa$ and $\alpha$ at $f=5-100 \mathrm{GHz}$ at $T=20^{\circ} \mathrm{C}$

\begin{tabular}{ccc}
\hline Frequencies $(\mathrm{GHz})$ & $\mathrm{k}$ & $\alpha$ \\
\hline 5 & 0.0048 & 3.3911 \\
10 & 0.3857 & 4.5272 \\
19.5 & 1.6169 & 4.2104 \\
25 & 2.4567 & 4.0186 \\
40 & 4.3106 & 3.5077 \\
60 & 6.0493 & 3.0094 \\
80 & 7.0623 & 2.6621 \\
100 & 7.6874 & 2.4156 \\
\hline
\end{tabular}

\section{RESULTS AND DISCUSSION}

\subsection{Specific Attenuation, Rainfall Rate and Frequencies}

Although various methods have been adopted by a number of researchers to investigate the particular contributions of certain drop diameters to the specific rain attenuation, our approach is to estimate 
the specific rain attenuation by integrating the raindrop size distribution $N(D)$ and the estinction cross section $Q(t)$ over the raindrop diameters as indicated in equation (10). For instance, Lee et al. [16] and [17], in Singapore, Fiser [18], in the Czech Republic, Lam et al. [19] in Malaysia, and Marzuki et al. [20] in the Equatorial Indonesia concluded that small and medium-size drops contributed more to the rainfall attenuation as frequency increases. They also noted that the prevailing contribution to the specificattenuation is formed by raindrops of diameter not exceeding $2 \mathrm{~mm}$ especially at higher frequencies. The attenuation created by drops in the diameter intervals $0.1 \geq D \geq 7.0 \mathrm{~mm}$ at various frequencies of transmission is shown in Tables 2 . The total specific attenuation increases with increasing frequencies for all the rainfall regimes. The specific rain attenuation increases with increased rain rates with the thunderstorm having the highest attenuation. The highest and prevailing contribution to the specific attenuation occurs at $D \sim 2 \mathrm{~mm}$ for the stratiform (drizzle or widespread) and convective (shower or thunderstorm) rain types shown in Figures 1 and 2 for the gamma and lognormal models, respectively (see Tables 4 and 5). The highest and prevailing contribution to the specific attenuation for the drizzle and widespread rainfall types occurs in the diameter range $0.5 \mathrm{~mm} \leq D \leq$ $2.5 \mathrm{~mm}$ while that of shower and thunderstorm rain types occurs in the range $1.0 \mathrm{~mm} \leq D \leq 3.0 \mathrm{~mm}$ and $1.5 \mathrm{~mm} \leq D \leq 3.5 \mathrm{~mm}$ respectively.

\subsection{Influence of Raindrop Diameters on Specific Rain Attenuation}

The percentage contribution formed by raindrop diameter intervals to the overall rain attenuation for different rain types is illustrated in Table 3. The contribution of larger diameters as observed is insignificant to the total attenuation. For instance, the highest contribution in the diameter range $4.0 \mathrm{~mm} \leq D \leq 7.0 \mathrm{~mm}$ is $1.46 \%$ and $0.0005 \%$ at $f=100 \mathrm{GHz}$ respectively for the thunderstorm and drizzle rain types. The largest contributions to the specific attenuation are due to drop diameters not exceeding $2 \mathrm{~mm}$ for all rainfall regimes at all frequencies and this confirms the results obtained in Singapore, Indonesia and Czech Republic. Hence, the diameter ranges $0.5 \mathrm{~mm} \leq D \leq 2.5 \mathrm{~mm}$ are critical to attenuation in Durban being a coastal region characterized by drizzle rainfall type.

Table 2. Total Specific Rain Attenuation Formed by Raindrops in the Diameter Interval $0.1 \mathrm{~mm} \geq D \geq 7.0$

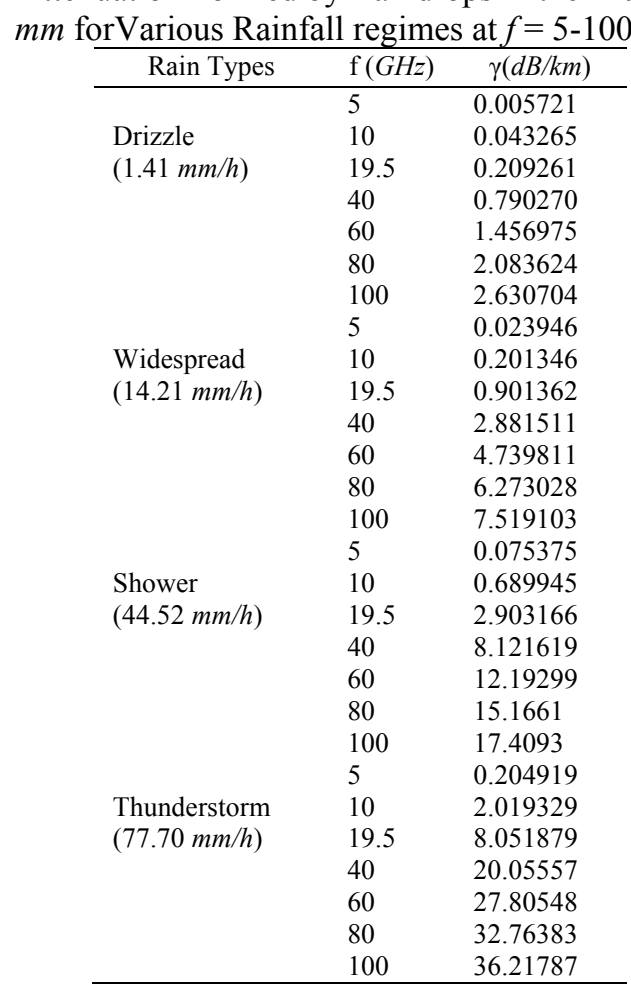


Table 3. Percentage $(\%)$ Contribution of the Specific Attenuation formed by Drop Diameters $(\mathrm{mm})$

\begin{tabular}{|c|c|c|c|c|c|c|}
\hline $\begin{array}{c}\text { Rain } \\
\text { Types }\end{array}$ & $\mathrm{f}(G H z)$ & $0.1 \leq D \leq 2$ & $0.5 \leq D \leq 2.5$ & $1.0 \leq D \leq 3.0$ & $1.5 \leq D \leq 3.5$ & $4.0 \leq D \leq 7.0$ \\
\hline \multirow{6}{*}{ Drizzle } & 5 & 95.55 & 99.25 & 81.93 & 29.99 & 0.004 \\
\hline & 10 & 94.21 & 98.95 & 85.05 & 34.59 & 0.007 \\
\hline & 19.5 & 95.21 & 99.18 & 82.82 & 31.21 & 0.005 \\
\hline & 40 & 96.95 & 99.51 & 77.09 & 24.29 & 0.002 \\
\hline & 60 & 97.83 & 99.64 & 72.43 & 19.94 & 0.001 \\
\hline & 80 & 98.31 & 99.69 & 68.90 & 17.20 & 0.0007 \\
\hline \multirow{8}{*}{ Widespread } & 100 & 98.59 & 99.71 & 66.29 & 15.41 & 0.0005 \\
\hline & 5 & 79.42 & 93.44 & 92.77 & 60.22 & 0.192 \\
\hline & 10 & 75.26 & 91.49 & 93.37 & 65.17 & 0.296 \\
\hline & 19.5 & 78.33 & 92.95 & 92.98 & 61.60 & 0.216 \\
\hline & 40 & 84.29 & 95.49 & 91.14 & 53.18 & 0.105 \\
\hline & 60 & 87.78 & 96.79 & 88.99 & 46.99 & 0.061 \\
\hline & 80 & 75.16 & 97.50 & 87.09 & 42.69 & 0.041 \\
\hline & 100 & 91.19 & 97.92 & 85.53 & 39.67 & 0.031 \\
\hline \multirow{6}{*}{ Shower } & 5 & 58.01 & 80.41 & 90.08 & 76.64 & 1.505 \\
\hline & 10 & 52.26 & 76.14 & 88.19 & 79.28 & 2.150 \\
\hline & 19.5 & 56.45 & 79.29 & 89.63 & 77.44 & 1.662 \\
\hline & 40 & 65.44 & 85.34 & 91.61 & 71.89 & 0.906 \\
\hline & 60 & 71.35 & 88.83 & 92.00 & 66.90 & 0.571 \\
\hline & 80 & 75.16 & 90.88 & 91.79 & 63.01 & 0.408 \\
\hline \multirow{8}{*}{ Thunderstorm } & 100 & 77.70 & 92.15 & 91.38 & 60.09 & 0.319 \\
\hline & 5 & 38.86 & 63.28 & 79.76 & 80.28 & 5.46 \\
\hline & 10 & 32.99 & 57.46 & 75.54 & 79.81 & 7.37 \\
\hline & 19.5 & 37.06 & 61.72 & 78.67 & 80.25 & 5.93 \\
\hline & 40 & 46.58 & 70.61 & 84.43 & 79.31 & 3.57 \\
\hline & 60 & 53.48 & 76.28 & 87.42 & 77.08 & 2.41 \\
\hline & 80 & 58.25 & 79.84 & 88.95 & 74.80 & 1.80 \\
\hline & 100 & 61.56 & 82.17 & 89.74 & 72.84 & 1.46 \\
\hline
\end{tabular}

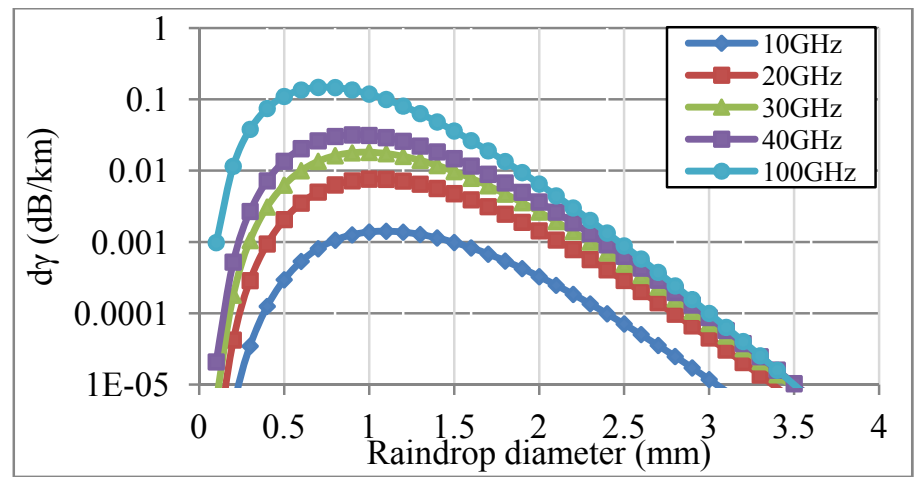

(a)

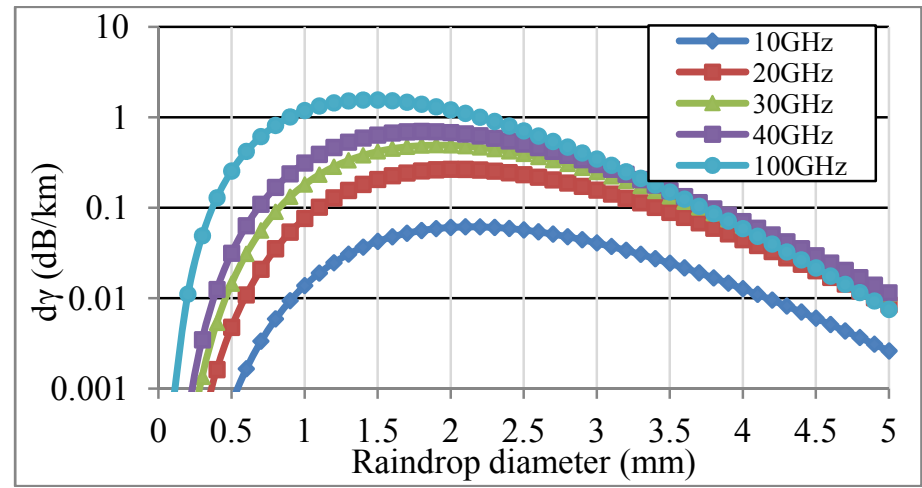

(b)

Figure 1. Rainfall attenuation and raindrop diameters for rainfall regimes at various frequencies for (a) $\mathrm{R}=$ 1.41 and (b) $\mathrm{R}=77.70 \mathrm{~mm} / \mathrm{h}$ using gamma DSD model 


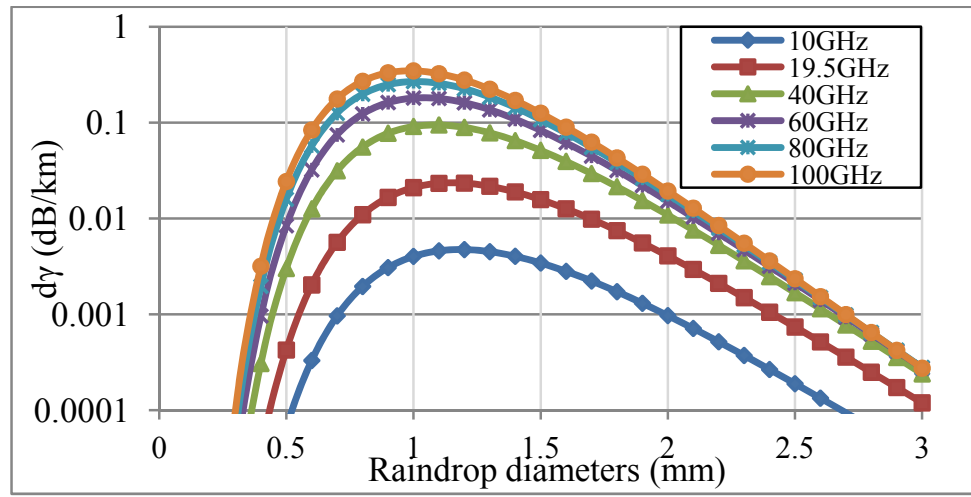

(a)

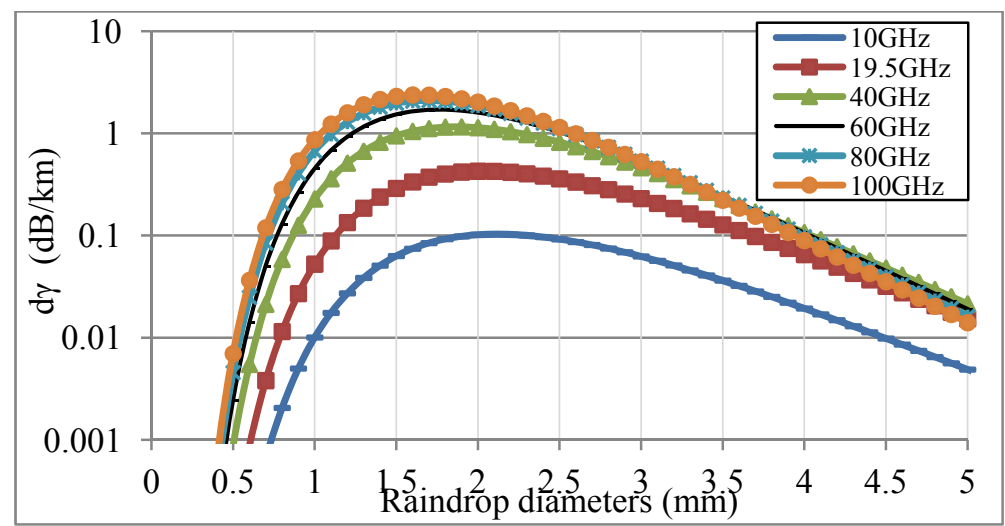

(b)

Figure 2. Rainfall attenuation and raindrop diameters for rainfall regimes at various frequencies for (a) $\mathrm{R}=1.41 \mathrm{~mm} / \mathrm{h}$ and (b) $\mathrm{R}=120 \mathrm{~mm} / \mathrm{h}$ using lognormal DSD model.

Table 4. Rain attenuation created by drops in the diameter range $0.1 \mathrm{~mm} \geq \mathrm{D} \geq 7.0 \mathrm{~mm}$ at various frequencies for the gamma model

\begin{tabular}{ccccc}
\hline $\begin{array}{c}\text { Frequency } \\
(\mathrm{GHz})\end{array}$ & Drizzle & Widespread & Shower & Thunderstorm \\
\hline 10 & 0.015 & 0.199 & 0.707 & 1.311 \\
20 & 0.081 & 0.925 & 3.079 & 5.533 \\
30 & 0.186 & 1.844 & 5.723 & 9.943 \\
40 & 0.318 & 2.781 & 8.111 & 13.670 \\
100 & 1.336 & 7.624 & 18.032 & 27.440 \\
\hline
\end{tabular}

Table 5. Rain attenuation created by drops in the diameter range $0.1 \mathrm{~mm} \geq \mathrm{D} \geq 7.0 \mathrm{~mm}$ at various frequencies for the lognormal model

\begin{tabular}{ccccc}
\hline $\begin{array}{c}\text { Frequency } \\
(\mathrm{GHz})\end{array}$ & Drizzle & Widespread & Shower & Thunderstorm \\
\hline 10 & 0.043 & 0.201 & 0.689 & 2.019 \\
19.5 & 0.209 & 0.901 & 2.903 & 8.051 \\
40 & 0.790 & 2.881 & 8.121 & 20.055 \\
60 & 1.457 & 4.739 & 12.192 & 27.805 \\
80 & 2.083 & 6.273 & 15.166 & 32.763 \\
100 & 2.630 & 7.519 & 17.409 & 36.217 \\
\hline
\end{tabular}

\section{CONCLUSION}

This paper considered the critical range of raindrop diameters at which the specific rainfall attenuation is most influenced. For the DSD models considered, the total percentage fraction formed by raindrops in the diameter range $0.5 \mathrm{~mm} \leq D \leq 2.5 \mathrm{~mm}$ and $1.0 \mathrm{~mm} \leq D \leq 3.0 \mathrm{~mm}$ are respectively found to be 
most critical for the specific rain attenuation for the stratiform (drizzle and widespread) and convective (shower and thunderstorm) in Durban, South Africa.

The contribution of larger diameters to the total attenuation is very low and insignificant when compared to medium and smaller diameters. The critical diameters are the range of diameters that contribute significantly to the rain attenuation. The highest contribution of raindrops diameters to the specific rain attenuation was created by drop diameters not exceeding $2 \mathrm{~mm}$, especially at higher frequencies. This confirms the results obtained by [16], [17] in Singapore; [18] in Czech Republic; [19] in Malaysia and [20] in Equatorial Indonesia. At frequency above $40 \mathrm{GHz}$, the drop size diameter that gives the largest contribution to the total attenuation for all the rain rates considered does not exceed $3 \mathrm{~mm}$ (90\%). This is similar to the results obtained by [20]. A good understanding of this rainfall attenuation characteristic will be helpful to properly design adequate fade margin levels, achieve the expected quality of service in a radio communication system operating in this region and for the purpose of link budget design by the engineers and service providers in this particular region.

\section{REFERENCES}

[1] H. Mohamed, A. Adel and A. Mohammed, "Rain measurements for MW-Wave Propagation: A Review", J. Eng. Sci., vol.11, no. 2, pp. 179-200, 1985

[2] Radiowave Propagation Series ITU, "Specific attenuation model for rain use in prediction models", ITU-R Recommendation P.838-1, 1999

[3] A.I. Yussuff and N.H. Khamis, "Rain Attenuation Modelling and Mitigation in the Tropics: Brief Review", International Journal of Electrical and Computer Engineering (IJECE). vol. 2, no. 6,pp. 748-757. 2012

[4] A.I. Yussuff, N.H. Khamis and A. Yahya "Performance Evaluation of Rain Attenuation Models in aTropical Station", International Journal of Electrical and Computer Engineering (IJECE). vol. 4, no. 5, pp. 782-789. 2014

[5] O.O. Obiyemi, O. Adetan and T.S. Ibiyemi, "Recent update on one-minute rainfall rate measurements for microwave applications in Nigeria", International Journal of Wireless Communications and Mobile Computing. vol. 3, pp. 33-39. 2015

[6] J.S. Marshall and W.M. Palmer, "The distribution of raindrops with size", Journal of meteorology, vol. 5, pp. 165$166,1948$.

[7] J.O. Laws and D.A. Parsons, "The relation of raindrops size to intensity", Eos, Transactions American Geophysical Union, vol. 24, pp. 452-460, 1943.

[8] D. Atlas and C.W. Ulbrich, "The Physical basis for attenuation-rainfall relationships and the measurement of rainfall parameters by combined attenuation and radar methods", J. Rech. Atmos., vol. 8, pp.275-298, 1974.

[9] G.O. Ajayi and R.L. Olsen, "Modeling of a tropical raindrop size distribution for microwave and millimeter wave applications", Radio Science. vol. 20, no 2, pp.193-202, 1985.

[10] O. Adetan and T.J. Afullo, "Comparison of two methods to evaluate the lognormal raindrop size distribution model in Durban", in proceedings of The SouthernAfrica Telecommunication Networks and Applications Conference (SATNAC), 2012, pp. 2-5.

[11] P. Owolawi, "Raindrop size distribution model for the prediction of rain attenuation in Durban", PIERS Online, vol. 7, no.6, pp.516-523, 2011.

[12] A.A. Alonge and T.J. Afullo, "Seasonal analysis and prediction of rainfall effects in Eastern South Africa at microwave frequencies", PIERS B, vol.40, pp. 279-303, 2012.

[13] M.O. Odedina and T.J. Afullo, "Determination of rain attenuation from electromagnetic scattering by spherical raindrops: Theory and experiment", Radio Science. vol. 45, 2010.

[14] T.J.O. Afullo, "Raindrop size distribution modeling for radio link along the Eastern coast of South Africa", PIERS $B$, vol.34, pp.345-366, 2011.

[15] O. Adetan and T.J. Afullo, "Three-parameter raindrop size distribution modeling for microwave propagation in South Africa", Proceedings of The International Association of Science and Technology for Development (IASTED), International Conference on Modeling and Simulation (Africa MS 2012), 2012,pp. 155-160.

[16] Y.H. Lee, S. Lakshmi and J.T. Ong, "Rain drop size distribution modeling in Singapore-critical diameters", in proceedings of the Second European Conference on Antennas and Propagation (EUCAP), 2007, pp. 1-5.

[17] S. Lakshmi, Y.H. Lee and J.T. Ong, "The roles of particular raindrop size on rain attenuation at $11 \mathrm{GHz}$ ", In Proceedings of 6th IEEE International Conference on Information, Communications \& Signal Processing (ICICS), 2007, pp.1-4.

[18] O. Fiser, "The role of particular rain drop size classes on specific rain attenuation at various frequencies with Czech data example", Proceedings of ERAD, vol. 113.no. 116, pp. 113-117, 2002.

[19] H.Y. Lam, J. Din, L. Luini, A.D. Panagopoulos and C. Capsoni, "Analysis of raindrop size distribution characteristics in Malaysia for rain attenuation prediction", In General Assembly and Scientific Symposium, 2011 XXXth URSI, 2011, pp. 1 - 4.

[20] M. Marzuki, T. Kozu, T. Shimomai, W.L. Randeu, H. Hashiguchi and Y. Shibagaski, "Diurnal variation of rain attenuation obtained from measurement of raindrop size distribution in Equatorial Indonesia", IEEE Transactions on Antennas and Propagation, vol. 57, no. 4, pp. 1191-1196, 2009.

[21] I.A. Adimula and G. O. Ajayi, "Variation in raindrop size distribution and specific attenuation due to rain in Nigeria", Annals of Telecommunications, vol. 51, no. 1-2, pp. 87-93, 1996. 
[22] M. J. Bartholomew, "Disdrometer and tipping bucket rain gauge handbook", ARM Climate Research Facility, 2009.

[23] R. Gunn and G.D. Kinzer, "Terminal velocity of fall for water drops in stagnant air", Journal of Applied Meteorology, vol. 6, pp.243-248, 1949.

[24] A. Tokay and D.A. Short, "Evidence from tropical raindrop spectra of the origin of rain from stratiform to convective clouds", Journal of Applied Meteorology, vol. 35, no. 3, pp. 355-371, 1996.

[25] C. Mätzler, "Drop-size distributions and Mie computation", IAP Research Report 2002-16, University of Bern, Bern, 2002.

[26] H.C. Van de Hulst., "Light Scattering by Small Particles", John Wiley and Sons Inc., New York, 1957.

[27] C.F. Bohren and D.R. Huffman, "Absorption and Scattering of Light by Small Particles", John Wiley \& Sons, 2008.

\section{BIOGRAPHIES OF AUTHORS}

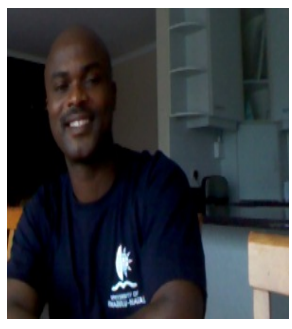

ADETAN Oluwumi holds B.Eng. degree in Electrical and Electronic Engineering from the then Ondo State University, Ado Ekiti, Nigeria in 1999; M.Eng. degree in Electrical and Electronic Engineering (Communications Option) from the Federal University of Technology, Akure, Nigeria in 2010 and Ph.D. degree in 2014 from the School of Electrical, Electronic and Computer Engineering, University of Kwazulu-Natal, South Africa. He joined the Department of Electrical and Electronic Engineering, Ekiti State University, Nigeria in 2000. He is a registered member the Institute of Electrical and Electronics Engineers (IEEE) and Council for the Regulation of Engineering in Nigeria (COREN). His research interest is to study the effects of rain on microwave propagation and wireless communications systems. He is currently the Acting Head of Department of Computer Engineering, Ekiti State University, Nigeria.

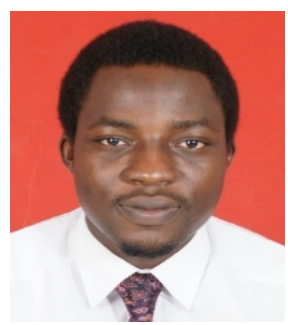

OBIYEMI Obiseyeobtained B.Eng. and M.Eng. Degree in Electrical Engineering from University of Ilorin, Nigeria in 2006 and 2010 respectively.He joined the Department of Electrical and Electronic Engineering, Osun State University in 2010 and $\mathrm{He}$ is currently workingtowards the $\mathrm{PhD}$ degree from the Department of Electrical and Electronic Engineering, University of Ilorin, Nigeria. 